\title{
Joint Resource Allocation for Lifetime-aware Cooperative Multi-carrier DF System
}

\author{
Chao-Ping Guo ${ }^{1,2}$ Kun Han ${ }^{1,2} \quad$ Xiao-Long Zhang ${ }^{1} \quad$ Hai-Lin Zhang ${ }^{1}$ \\ ${ }^{1}$ State Key Laboratory of Integrated Services Network, Xidian University, Xi'an 710071, China \\ ${ }^{2} \mathrm{Xi}^{\prime}$ an Communication Institute, $\mathrm{Xi}^{\prime}$ an 710106 , China
}

\begin{abstract}
In this paper, we study the problem of optimal resource allocation for lifetime maximization in an orthogonal-frequencydivision multiplexing (OFDM) system with decode-and-forward relay. The goal is to minimize total energy cost of the system by jointly optimizing power allocation, subcarrier pairing and relay selection. We present a heuristic solution that is composed of two parts. The first part is an optimal power allocation approach to allocate power to a subcarrier pair of the source and the relay. The second part is a modified Hungarian algorithm to make subcarrier pairing and relay selection. Evaluations show that the presented scheme outperforms other schemes in the total transmitted data and the network lifetime.
\end{abstract}

Keywords: Cooperative communications, resource allocation, decode-and-forward, orthogonal-frequency-division multiplexing (OFDM), subcarrier pairing, power allocation, relay selection.

\section{Introduction}

Cooperative relaying has been considered as a main area of research and development for next generation wireless networking. Meanwhile, orthogonal-frequency-division multiplexing (OFDM) has received more and more attentions because of its mitigating frequency selective fading by assigning bandwidth to parallel subcarriers. Therefore, $4 \mathrm{G}$ wireless systems propose OFDM-based relaying networking framework.

From a viewpoint of quality of services (QoS), the objective of the system is to maximize system capacity under power constraint ${ }^{[1-4]}$, or minimize transmission power to meet for QoS requirement ${ }^{[5,6]}$. These two resource allocation approaches may quickly exhaust the energy of a mobile wireless node and decrease network lifetime. While, a few studies so far focus on lifetime maximization for cooperative relaying system. In [7], a relay selection method is developed for lifetime extension in an amplify-and-forward (AF) cooperative network where the transmission power is subject to a signal-to-noise ratio (SNR) requirement at the destination. In [8], a relay selection and power allocation scheme is proposed for decode-and-forward relay (DF) cooperative network based on energy pricing. In [9], dynamic single relay selection and power allocation schemes are devised to prolong the lifetime of a single-source AF cooperative network. In [10], perceived lifetime power allocation strategy and minimum weighted total power strategy are proposed to prolong networks lifetime, without relay selection and power allocation for the source. In [11], predictionbased perceived lifetime power allocation algorithm for net-

\footnotetext{
Manuscript received September 9, 2012; revised April 16, 2013

This work was supported by Important National Science and Technology Specific Projects (No.2011ZX03003-001-04 and 2012ZX03003-012), National Natural Science Foundation of China (No. 61072069), Fundamental Research Funds for the Central Universities (No. 72101859), State Key Laboratory of Integrated Services Networks (No. ISN090105), and 111 Project (No. B08038)
}

work lifetime maximization is presented. In these existing prolonging lifetime schemes, source power allocation is not studied $^{[7,10,11]}$ and none of them are involved in subcarrier assignment.

In this paper, we make balanced use of energy of the system to extend the lifetime of a dual-hop cooperative OFDM system operated by energy constrained relays using DF protocol. We assume that the source and the relay have their individual power constraints because it is more practical. We first formulate the problem of total energy cost minimization as a total energy cost minimizing integer programming problem, which can be resolved by dual method. However, this poses a heavy computation burden on the node to perform resource allocation. Hence, we present a heuristic solution to reduce the complexity.

The paper is organized as follows. In Section 2, we introduce system model and formulate our problem. We present heuristic solution to our problem in Section 3. We first derive optimal power allocation solution for a subcarrier pair of the source and the relay, and then present a modified Hungarian algorithm to make subcarrier pairing and relay selection. In Section 4, simulations are presented to show the performance of the resource allocation scheme. Section 5 concludes the paper.

\section{System model and problem formula- tion}

\subsection{System model}

Consider a dual-hop half-duplex DF relay cooperation OFDM system, consisting of a source $S$, a relay $R$ and a destination $D$. The total frequency band is divided into $N$ subcarriers. OFDM with the same spectral occupancy is used for all links. The transmission is completed in two time slots and each of them is of the same duration $T$. In the first slot, the source transmits the signals on all subcar- 
riers while the destination and the relay listen. In the second slot, the source remains silent while the relay decodes, encodes and forwards the received signals on a subcarrier basis, and forwards them to the destination. Suppose that the relay decodes the signal transmitted from the source and decodes it with the same codebooks as the one used by the source. Assume that subcarrier $k$ in the first time slot and subcarrier $m$ in the second time slot are paired and they form a subcarrier pair $S P(k, m)$. Therefore, the number of subcarrier pairs in transmission is $N$. The destination maximum ratio combines (MRC) the signals from the source in the first time slot and the signals from the relay in the second time slot pertaining to the same message to exploit the spatial diversity. The messages transmitted on different subcarrier pairs are assumed to be independent. The channel fading coefficients are assumed to remain constant in a two-slot period and all the channel parameters are assumed known at the destination which will perform resource assignment. The destination then informs the relay and the source of the corresponding parameters via control channel before the data transmission.

For subcarrier pair $S P(k, m)$, we use $h_{s d}(k)$ to denote the channel gains of the SD link on subcarrier $k, h_{s r}(k)$ to denote the channel gains of that of SR link on subcarrier $k$, and $h_{r d}(m)$ to denote the channel gains of RD link on subcarrier $m$, respectively. $\delta_{s d}^{2}(k), \delta_{s r}^{2}(k)$ and $\delta_{r d}^{2}(m)$ are the variances of the additive white Gaussian noises (AWGN) in the corresponding channels. $W$ is the transmission bandwidth of each subcarrier. We use $\gamma_{s d}^{2}(k)=\frac{\left|h_{s d}^{2}(k)\right|^{2} N}{\left(\delta_{s d}^{2}(k) W\right)}$, $\gamma_{s r}^{2}(k)=\frac{\left|h_{s r}^{2}(k)\right|^{2} N}{\left(\delta_{s r}^{2}(k) W\right)}$ and $\gamma_{r d}^{2}(m)=\frac{\left|h_{r d}^{2}(m)\right|^{2} N}{\left(\delta_{r d}^{2}(m) W\right)}$ to denote the normalized channel gains. Let $p_{s}(k)$ denote the transmission power of the source on subcarrier $k$ and $p_{r}(m)$ denote the transmit power of the relay on subcarrier $m$, respectively. When the source uses subcarrier $k$ to transmit signals directly, the attainable data rate can be expressed as

$$
R^{\mathrm{dir}}(k)=0.5 W \log _{2}\left(1+\gamma_{s d}(k) p_{s}(k)\right)
$$

When the source uses subcarrier pair $S P(k, m)$ to transmit signals, the attainable data rate can be written as

$$
\begin{gathered}
R^{\mathrm{coop}}(k, m)=\min \left\{0.5 W \log _{2}\left(1+\gamma_{s r}(k) p_{s}(k)\right),\right. \\
\left.0.5 W \log _{2}\left(1+\gamma_{s d}(k) p_{s}(k)+\gamma_{r d}(m) p_{r}(m)\right)\right\} .
\end{gathered}
$$

Under the power constraint of the source and the relay, the relay is used when ${ }^{[1]}$

$$
\begin{aligned}
& \gamma_{s r}(k) p_{s}(k) \geqslant \gamma_{s d}(k) p_{s}(k) \quad \text { and } \\
& \gamma_{r d}(m) p_{r}(m) \geqslant \gamma_{s d}(k) p_{s}(k) .
\end{aligned}
$$

\subsection{Problem formulation}

In order to make balanced use of energy of the system, we now present a resource allocation scheme based on energy pricing. We model the source and the relay as energy sellers. From a supply and demand perspective, we can conclude that the nodes which have less residual energy should charge a higher price, and vice versa. The source buys the energy from the relay in order to fulfill the transmission of the data block with minimum cost. We assume that $\rho_{s}$ and $\rho_{r}$ be the energy price of the source, the energy price of the relay, respectively. The energy cost paid by the user is $\rho_{s} p_{s}(k) T$ when the subcarrier $k$ of the source is used to transmit signals for duration $T$. The energy cost paid by the user is $\rho_{r} p_{r}(m) T$ when the subcarrier $m$ of the relay is used to transmit signals for duration $T$. To unify system model, we also use $S P(k, 0)$ to denote direct transmission on subcarrier $k$ of the source $S$. The objective is to minimize the total energy cost of the source and the relay to meet the rate requirement $R(k)$ of each subcarrier under their power constraint $P$. The total energy cost minimization problem can be formulated as

$$
\begin{gathered}
\min \sum_{k}\left\{t(k, 0) \rho_{s} p_{s}(k) T+\sum_{m} t(t, m) T\left[\rho_{s} p_{s}(k)+\rho_{r} p_{r}(m)\right]\right\} \\
\text { s.t. } t(k, 0) R^{\mathrm{dir}}(k)+t(k, m) R^{\mathrm{coop}}(k, m) \geqslant R(k) \\
\sum_{m=0}^{N} t(k, m)=1, \quad \forall k \\
\quad \sum_{k=1}^{N} t(k, m)=1, \quad \forall m>0 \\
t(k, m)=\{0,1\}, \quad \forall k \text { and } \forall m \\
\sum_{k=1}^{N} p_{s}(k) \leqslant P \\
\sum_{m=1}^{N} p_{r}(m) \leqslant P \\
p_{s}(k) \geqslant 0, \quad \forall k, \text { and } p_{r}(m) \geqslant 0, \quad \forall m
\end{gathered}
$$

where we define an indicator as $t(k, m)$ which is 1 if subcarrier $k$ of the source is paired with subcarrier $m$ and 0 otherwise. The constraint (5) indicates that for any subcarrier $k$, the rate requirement $R(k)$ must be satisfied by either direct transmission or relay cooperation. The constraint (6) means that the source uses subcarrier to convey signals either directly or together with only one subcarrier $m$ of the relay. The constraint (7) indicates that subcarrier $m$ of the relay is paired with at most one subcarrier of the source to transmit signals. The constraints (9) and (10) are individual power constraints of the source and the relay respectively.

\section{Joint resource allocation scheme based on energy pricing}

Obviously, equations (4)-(11) form an integer programming problem. The complexity grows exponentially when the number of the subcarriers increases. The destination has to recompute optimal power, subcarrier allocation, and relay decisions for both the relay and the source as the channel changes. Hence, fast and low complexity suboptimal algorithms are more reasonable options to reduce the computational burden of the destination.

In order to find a suboptimal solution, we divide our optimization problem in two parts. The first part is to find optimal power allocation for an arbitrary subcarrier pair of 
the source and the relay. We use graphic method to obtain it. The second part is to obtain subcarrier pairing and relay selection by combining Hungarian algorithm ${ }^{[12]}$ and greedy algorithm.

\subsection{Optimal power allocation}

In this section, we derive optimal power allocation solution for subcarrier pair $S P(k, m)$ based on graphic method. We assume that $P_{\text {tot }}^{s}$ is the power constraint of the source and $P_{\text {tot }}^{r}$ is the power constraint of the relay, and $R(k)$ is the data rate requirement of subcarrier $k$ of the source. We also assume subcarrier $k$ of the source is paired with subcarrier $m$ of the relay. For subcarrier pair $S P(k, m)$, the problem of optimal power allocation can be formulated as

$$
\begin{gathered}
\min \left\{C=\rho_{s} p_{s}(k) T+\rho_{r} p_{r}(m) T\right\} \\
\gamma_{s d}(k) p_{s}(k)+\gamma_{r d}(m) p_{r}(m) \geqslant 2^{\frac{2 R(k)}{W}}-1 \\
p_{s}(k) \geqslant p_{s}^{\text {low }}(k) \\
p_{s}(k) \leqslant P_{\text {tot }}^{s} \\
p_{r}(m) \leqslant P_{\text {tot }}^{r}
\end{gathered}
$$

where $p_{s}^{\text {low }}(k)=\frac{2^{\frac{2 R(k)}{W}}-1}{\gamma_{s r}(k)}$. Define rate function $f\left(p_{s}(k), p_{r}(m)\right)=0.5 W^{\gamma^{\prime} \log _{2}}\left(\gamma_{s d}(k) p_{s}(k)+\gamma_{r d}(m) p_{r}(m)\right)$. The solution to (12)-(16) is shown in Fig. $1^{[8]}$, where the lines $S_{i}(i=1,2,3)$ denote the SNR line $\gamma_{s d}(k) p_{s}(k)+$ $\gamma_{r d}(m) p_{r}(m)=2^{\frac{2 R(k)}{W}}-1$ when $R(k)$ takes different values. Let the cost lines $C_{1}$ and $C_{2}$ denote the cost line $C=$ $\rho_{s} p_{s}(k) T+\rho_{r} p_{r}(m) T$ when $\frac{\rho_{s}}{\rho_{r}}>\frac{\gamma_{s d}(k)}{\gamma_{r d}(m)}$ and $\frac{\rho_{s}}{\rho_{r}} \leqslant \frac{\gamma_{s d}(k)}{\gamma_{r d}(m)}$ respectively. The shaded region represents the points of $\left(p_{s}(k), p_{r}(m)\right)$ satisfying the constraints (13)-(16). In this paper, the case $\frac{\rho_{s}}{\rho_{r}}=\frac{\gamma_{s d}(k)}{\gamma_{r d}(m)}$ omitted in [8] is considered. The first intersection point of $C_{i}(i=1,2,3)$ and the shaded region satisfies the given constraint conditions (13)-(16) and takes the minimum cost, which is exactly the optimal power allocation solution. When $p_{s}^{\text {low }}(k)>P_{\text {tot }}^{s}$ or $R(k)>f\left(P_{\mathrm{tot}}^{s}, P_{\mathrm{tot}}^{r}\right)$, there does not exist the optimal solution. In the following, we discuss the optimal solution under the conditions $p_{s}^{\text {low }}(k) \leqslant P_{\text {tot }}^{s}$ and $R(k) \leqslant f\left(P_{\text {tot }}^{s}, P_{\text {tot }}^{r}\right)$, which can be divided into three cases:

1) $\frac{\rho_{s}}{\rho_{r}}>\frac{\gamma_{s d}(k)}{\gamma_{r d}(m)}$ case. If $f\left(p_{s}^{\text {low }}(k), 0\right) \leqslant R(k) \leqslant f\left(p_{s}^{\text {low }}(k)\right.$, $\left.P_{\text {tot }}^{r}\right)$, optimal power allocation lies in point $d_{3}$, i.e., $p_{s}(k)=p_{s}^{\text {low }}(k)$ and $p_{r}(m)=h_{2}\left(p_{s}^{\text {low }}(k), \gamma_{s d}(k), \gamma_{r d}(m)\right)$. If $f\left(p_{s}^{\text {low }}(k), P_{\text {tot }}^{r}\right) \leqslant R(k) \leqslant f\left(P_{\text {tot }}^{s}, P_{\text {tot }}^{r}\right)$, optimal power allocation lies in point $d_{1}$, i.e., $p_{r}(m)=P_{\text {tot }}^{r}$ and $p_{s}(k)=$ $h_{1}\left(P_{\text {tot }}^{r}, \gamma_{s d}(k), \gamma_{r d}(m)\right)$.

2) $\frac{\rho_{s}}{\rho_{r}}<\frac{\gamma_{s d}(k)}{\gamma_{r d}(m)}$ case. If $f\left(p_{s}^{\text {low }}(k), 0\right) \leqslant R(k) \leqslant f\left(P_{\text {tot }}^{s}, 0\right)$, optimal power allocation lies in point $d_{6}$, i.e., $p_{s}(k)=$ $h_{1}\left(0, \gamma_{s d}(k), \gamma_{r d}(m)\right)$ and $p_{r}(m)=0$. If $f\left(P_{\text {tot }}^{s}, 0\right) \leqslant R(k) \leqslant$ $f\left(P_{\text {tot }}^{s}, P_{\text {tot }}^{r}\right)$, optimal power allocation lies in point $d_{2}$, i.e., $p_{s}(k)=P_{\text {tot }}^{s}$ and $p_{r}(m)=h_{2}\left(P_{\mathrm{tot}}^{s}, \gamma_{s d}(k), \gamma_{r d}(m)\right)$.

3) $\frac{\rho_{s}}{\rho_{r}}=\frac{\gamma_{s d}(k)}{\gamma_{r d}(m)}$ case. There are two subcases:

a) $f\left(P_{\text {tot }}^{s}, 0\right) \leqslant f\left(p_{s}^{\text {low }}(k), P_{\text {tot }}^{r}\right)$ subcase. If $f\left(p_{s}^{\text {low }}(k)\right.$, $0) \leqslant R(k) \leqslant f\left(P_{\text {tot }}^{s}, 0\right)$, then $p_{s}(k) \in\left[p_{s}^{\text {low }}(k), h_{1}(0\right.$, $\left.\left.\gamma_{s d}(k), \gamma_{r d}(m)\right)\right]$ and $p_{r}(m)=h_{2}\left(p_{s}(k), \gamma_{s d}(k), \gamma_{r d}(m)\right)$. If $f\left(P_{\mathrm{tot}}^{s}, 0\right) \leqslant R(k) \leqslant f\left(p_{s}^{\text {low }}(k), P_{\mathrm{tot}}^{r}\right)$, then $p_{s}(k) \in$ $\left[p_{s}^{\text {low }}(k), P_{\text {tot }}^{s}\right]$ and $p_{r}(m)=h_{2}\left(p_{s}(k), \gamma_{s d}(k), \gamma_{r d}(m)\right)$. If $f\left(p_{s}^{\text {low }}(k), P_{\text {tot }}^{r}\right) \leqslant R(k) \leqslant f\left(P_{\text {tot }}^{s}, P_{\text {tot }}^{r}\right)$, then $p_{s}(k) \in$ $\left[h_{1}\left(P_{\text {tot }}^{r}, \gamma_{s d}(k), \gamma_{r d}(m)\right), P_{\text {tot }}^{s}\right]$ and $p_{r}(m)=h_{2}\left(p_{s}(k)\right.$, $\left.\gamma_{s d}(k), \gamma_{r d}(m)\right)$.

b) $f\left(P_{\text {tot }}^{s}, 0\right)>f\left(p_{s}^{\text {low }}(k), P_{\text {tot }}^{r}\right)$ subcase. If $f\left(p_{s}^{\text {low }}(k)\right.$, $0) \leqslant R(k) \leqslant f\left(p_{s}^{\text {low }}(k), P_{\text {tot }}^{r}\right)$, then $p_{s}(k) \in\left[p_{s}^{\text {low }}(k), h_{1}(0\right.$, $\left.\left.\gamma_{s d}(k), \gamma_{r d}(m)\right)\right]$ and $p_{r}(m)=h_{2}\left(p_{s}(k), \gamma_{s d}(k), \gamma_{r d}(m)\right)$. If $f\left(p_{s}^{\text {low }}(k), \quad P_{\text {tot }}^{r}\right) \leqslant R(k) \leqslant f\left(P_{\text {tot }}^{s}, 0\right)$, then $p_{s}(k) \in\left[h_{1}\left(P_{\text {tot }}^{r}, \gamma_{s d}(k), \gamma_{r d}(m)\right), h_{1}\left(0, \gamma_{s d}(k), \gamma_{r d}(m)\right)\right]$ and $p_{r}(m)=h_{2}\left(p_{s}(k), \gamma_{s d}(k), \gamma_{r d}(m)\right)$. If $f\left(P_{\text {tot }}^{s}, 0\right) \leqslant R(k) \leqslant$ $f\left(P_{\text {tot }}^{s}, P_{\text {tot }}^{r}\right)$, then $p_{s}(k) \in\left[h_{1}\left(P_{\text {tot }}^{r}, \gamma_{s d}(k), \gamma_{r d}(m)\right), P_{\text {tot }}^{s}\right]$ and $p_{r}(m)=h_{2}\left(p_{s}(k), \gamma_{s d}(k), \gamma_{r d}(m)\right)$.

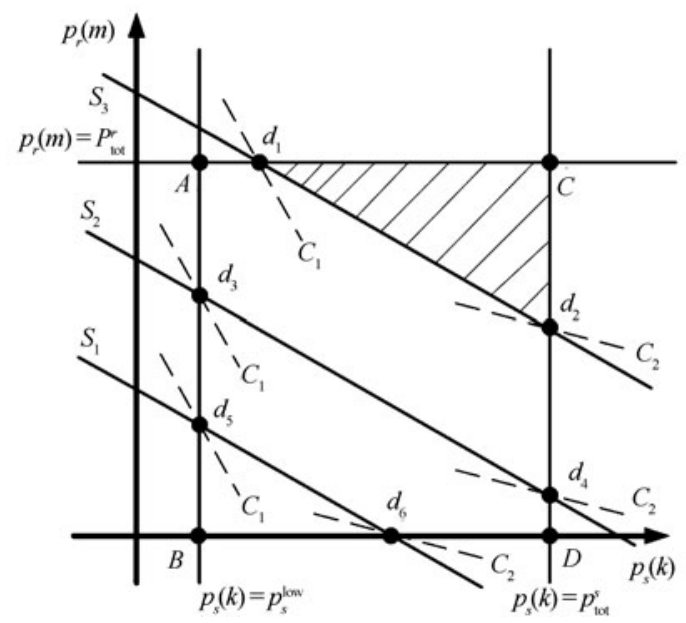

Fig. 1 The optimal power allocation model for $S P(k, m)$

We define function $h_{1}(x, y, z)=\frac{2^{\frac{2 R(k)}{W}}-x z-1}{y}$ and $h_{2}(x, y, z)=\frac{2^{\frac{2 R(k)}{W}-x y-1}}{z}$. With the optimal power allocation, the energy cost for $S P(k, m)$ can be computed with $C=\rho_{s} p_{s}(k) T+\rho_{r} p_{r}(m) T$.

\subsection{Modified Hungarian algorithm}

For the source's subcarrier with the energy cost of direct transmission being not larger than the minimum of the energy cost of relay modes, we let it be operated in direct transmission mode. And for all of the source's other subcarriers, we let them be operated in relay mode. Then, we combine Hungarian algorithm with greedy algorithm to make subcarrier pairing and relay selection for the source's subcarrier operated in relay mode.

For subcarrier $k$ of the source, the power allocated to direct transmission and the energy cost of direct transmission are

$$
\begin{aligned}
& p_{s}^{\mathrm{dir}}(k)=\frac{2^{\frac{2 R(k)}{W}}-1}{\gamma_{s d}(k)} \\
& C^{\mathrm{dir}}(k)=\rho_{s} p_{s}^{\mathrm{dir}}(k) T .
\end{aligned}
$$

Based on this, modified Hungarian algorithm is designed as Algorithm 1. 
Algorithm 1. Modified Hungarian algorithm

1) Initialize $R^{\text {avi }}(k)=\{1,2, \cdots, N\}, p_{s}^{\text {temp }}, p_{r}^{\text {temp }}$ and $C^{\text {temp }}$ as a $N \times N$ array with every element to be $10^{5}$, respectively, $N_{c}=0, I_{c}=\{0,0, \cdots, 0\}$.

2) For $k=1,2, \cdots, N$

Use (17) and (18) to compute the power $p_{s}^{\text {dir }}(k)$ and the energy cost $C^{\text {dir }}(k)$ of direct mode

If (3) does not hold

Move the subcarrier $m$ from its available subcarrier set $R^{\text {avi }}(k)$

end if

end for

3) For $k=1,2, \cdots, N$

For all $m \in R^{\text {avi }}(k)$

Obtain optimal power allocation for $S P(k, m)$ as

the method in Subsection 3.1 and let $p_{s}^{\text {temp }}(k, m)=$ $p_{s}(k), p_{r}^{\mathrm{temp}}(k, m)=p_{r}(m)$ and $C^{\mathrm{temp}}(k, m)=C$. end for

end for

4) for $k=1,2, \cdots, N$

$$
\begin{aligned}
& \text { if } C^{\text {dir }}(k)<\min \left\{C^{\text {temp }}(k,:)\right\} \\
& \quad p_{s}^{\text {out }}(k)=p_{s}^{\text {dir }}(k), p_{r}^{\text {out }}(m)=0, C^{\text {out }}(k)=C^{\operatorname{dir}}(k),
\end{aligned}
$$$$
\text { and } m(k)=0
$$

end if

end for

5) For $l=1,2, \cdots, N_{c}$

$C(l,:)=C^{\text {temp }}\left(I(l), 1: N_{c}\right)$

end for

6) Execute Hungarian algorithm with $C$ and obtain assignment result $Q$.

7) If $Q$ exists

$$
\text { for } l=1,2, \cdots, N_{c}
$$

Find $m$ such that $Q(l, m)=1, k=I(l)$

Compute $C_{1}^{\mathrm{min}}=\min \left\{C^{\mathrm{temp}}\left(k,\left(N_{c}+1\right): N\right)\right\}$ and $C_{2}^{\mathrm{min}}=\min \left\{C_{1}^{\mathrm{min}}, C^{\mathrm{temp}}(k, m), C^{\mathrm{dir}}(k)\right\}$.

if $C_{2}^{\min }=C^{\text {dir }}(k)$

$p_{s}^{\text {out }}(k)=p_{s}^{\text {dir }}(k), p_{r}^{\text {out }}(m)=0, C^{\text {out }}(k)=$

$C^{\text {dir }}(k)$ and $m(k)=0$.

else if $C_{2}^{\min }=C^{\text {temp }}(k, m)$

$$
p_{s}^{\text {out }}(k)=p_{s}^{\text {temp }}(k, m), p_{r}^{\text {out }}(m)=p_{r}^{\text {temp }}(k, m),
$$$$
C^{\text {out }}(k)=C^{\text {temp }}(k, m) \text {, and } m(k)=m \text {. }
$$

else

find $m^{\prime}$ such that $C^{\text {temp }}\left(k, m^{\prime}\right)=C_{1}^{\text {min }}$.

$p_{s}^{\text {out }}(k)=p_{s}^{\text {temp }}\left(k, m^{\prime}\right), p_{r}^{\text {out }}(m)=p_{r}^{\text {temp }}\left(k, m^{\prime}\right)$,

$C^{\text {out }}(k)=C^{\text {temp }}\left(k, m^{\prime}\right)$, and $m(k)=m^{\prime}$.

for $l^{\prime}=(l+1),(l+2), \cdots, N_{c}$

$k^{\prime}=I\left(l^{\prime}\right)$ and $C^{\text {temp }}\left(k^{\prime}, m^{\prime}\right)=10^{5}$.

end for

end if

end for

else

for $l=1,2, \cdots, N_{c} k=I(l)$.

Compute $C_{1}^{\text {min }}=\min \left\{C^{\text {temp }}(k,:)\right\}$ and find $m$

such that $C^{\text {temp }}(k, m)=C_{1}^{\text {min }}$.

$p_{s}^{\text {out }}(k)=p_{s}^{\text {temp }}(k, m), p_{r}^{\text {out }}(m)=p_{r}^{\text {temp }}(k, m)$,

$C^{\text {out }}(k)=C^{\text {temp }}(k, m)$, and $m(k)=m$.

for $l^{\prime}=(l+1),(l+2), \cdots, N_{c}$

$$
k^{\prime}=I\left(l^{\prime}\right) \text { and } C^{\text {temp }}\left(k^{\prime}, m\right)=10^{5} .
$$

end for

end for

end if

8) while $\sum_{k=1}^{N} p_{s}^{\text {out }}(k)>P_{\text {tot }}^{s}$ or $\sum_{m=1}^{N} p_{r}^{\text {out }}(m)>P_{\text {tot }}^{r}$ find $k$ such that $C^{\max }=\max \left\{C^{\text {out }}\left(k^{\prime}\right) \mid k^{\prime}=\right.$ $1,2, \cdots N\}$, let $p_{s}^{\text {out }}(k)=0, p_{r}^{\text {out }}(m)=0$, $C^{\text {out }}(k)=0$ and $m(k)=0$.

end while

Here $p_{s}^{\text {out }}(k)$ is the power allocated to subcarrier $k$ of the source, $m(k)$ is the index of the relay's subcarrier which is paired with subcarrier $k$ of the source, $p_{r}^{\text {out }}(k)$ is the power allocated to this subcarrier of the relay, and $C(k)$ is the energy cost of this subcarrier pair. In Step 7), if there exists the solution of Hungarian algorithm, we select the transmission mode corresponding to the minimum energy cost for subcarrier $k$ of the source, otherwise we use greedy algorithm to perform subcarrier pairing and relay selection. In Step 8), we do not allocate power to subcarrier pair $S P(k, m)$ with the largest energy cost if the allocated total power of the source exceeds its total power constraint, or the allocated total power of the relay exceeds its total power constraint.

\subsection{Complexity analysis}

In the method to obtain optimal power allocation, there needs 8 comparison operations, 17 multiplications, 5 additions and 2 logarithmic ones to obtain optimal power allocations.

An exhaustive search can be used to determine the optimal subcarrier pairing. There needs $N$ ! combination operations and $(N !-1)$ comparisons for the exhaustive search. The complexity of Hungarian algorithm is $\mathrm{O}\left(N^{3}\right)$ and the greedy algorithm needs $\frac{N(N+1)}{2}$ combination operations to obtain suboptimal subcarrier pairing. Hence, the complexity of Algorithm 1 is $\mathrm{O}\left(N^{3}\right)$.

\section{Evaluations}

In this section, we first present the definition of energy price and then present simulation results to show the performance of the presented scheme.

We assume $\rho_{0}$ be the initial energy price of the source (also the initial energy price of the relay), $\eta$ be the energy indicator which takes the value 1 or $2, E_{s}^{\text {tot }}$ be the initial energies of the source, $E_{r}^{\text {tot }}$ be the initial energies of the relay, $E_{s}^{\text {rem }}$ be the residual of the source, and $E_{r}^{\text {rem }}$ be the residual energy of the source. The energy price of the source and the energy price of the relay can be defined as ${ }^{[8]}$

$$
\begin{aligned}
& \rho_{s}=\rho_{0}\left(\frac{E_{s}^{\mathrm{tot}}}{E_{s}^{\mathrm{rem}}}\right)^{\eta} \\
& \rho_{r}=\rho_{0}\left(\frac{E_{r}^{\mathrm{tot}}}{E_{r}^{\mathrm{rem}}}\right)^{\eta} .
\end{aligned}
$$

In all simulations, the wireless channel ${ }^{[13]}$ is modeled as a frequency selective channel consisting of six independent Rayleigh multipaths. Each multipath component is 
modeled by Clarkes flat fading model. It is assumed that the power delay profile is exponentially decaying with $\mathrm{e}^{-\tau}$, where $\tau$ is the multipath index. Hence, the relative power of the six multipath components are $[0,-8.69,-17.37$, $-26.06,-34.74,-43.43] \mathrm{dB}$. The total available bandwidth and transmission power are $1 \mathrm{MHz}$ and $1 \mathrm{~W}$, respectively. Other parameters are $\sigma^{-2}=10^{-8}, N=64, \eta=2, \rho_{0}=10$, $E_{s}^{\text {tot }}=E_{r}^{\text {tot }}=1 \mathrm{~J}, T=10^{-1} \mathrm{~s}$. The definition of the network lifetime is the network operation time until the first node exhausts its energy ${ }^{[8]}$. The other two schemes minimum energy scheme and direct transmission scheme are also evaluated. By setting $\rho_{s}=\rho_{r}=1$, optimal power assignment approach based on graphic method becomes identical to minimum transmission energy algorithm, and we call this joint resource allocation scheme as minimum energy scheme. The simulation is executed 1000 times and the result is shown in Figs. 2 and 3.

From Fig. 2, we can see that network lifetime of these three schemes decrease as total data rate on all the subcarriers increases, and joint resource allocation scheme outperforms the other two schemes. From Fig. 3, we can see that total transmitted data of these three schemes decrease as total data rate on all the subcarriers increases, and modified Hungarian scheme outperforms the other two schemes. But total transmitted data of joint resource allocation scheme and minimum energy scheme are nearly 3 times that of direct mode scheme. The reason lies that minimum energy scheme selects transmit with the lowest energy mode from direct transmit mode and relay transmit mode, and the same situation exists when the nodes' energy is very low. Joint resource allocation scheme takes the residuary energy of the node into account, selects transmission mode with the lowest energy cost, and makes balanced use of energy of the system. So joint resource allocation scheme works better than minimum energy scheme in balanced energy use of the system.

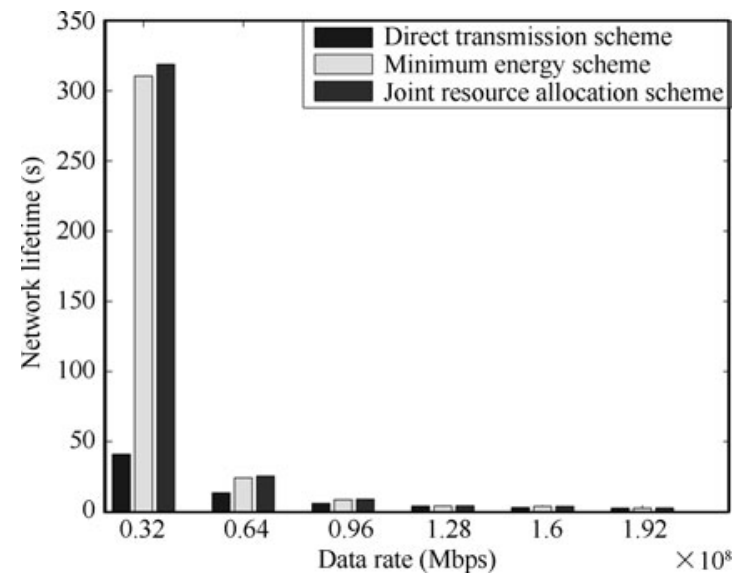

Fig. 2 The network lifetime versus total data rate

\section{Conclusions}

In this paper, we consider balanced energy usage of the system in an energy constrained cooperative OFDM system. The optimization problem takes the energy cost of the source and the energy cost of the relay into account. The proposed scheme consists of two parts: The first part is an optimal power allocation approach to allocate power to a subcarrier pair of the source and the relay. The second part is a modified Hungarian algorithm to make subcarrier pairing and relay selection. Evaluations show that the presented scheme outperforms other schemes in terms of the total transmitted data and the network lifetime.

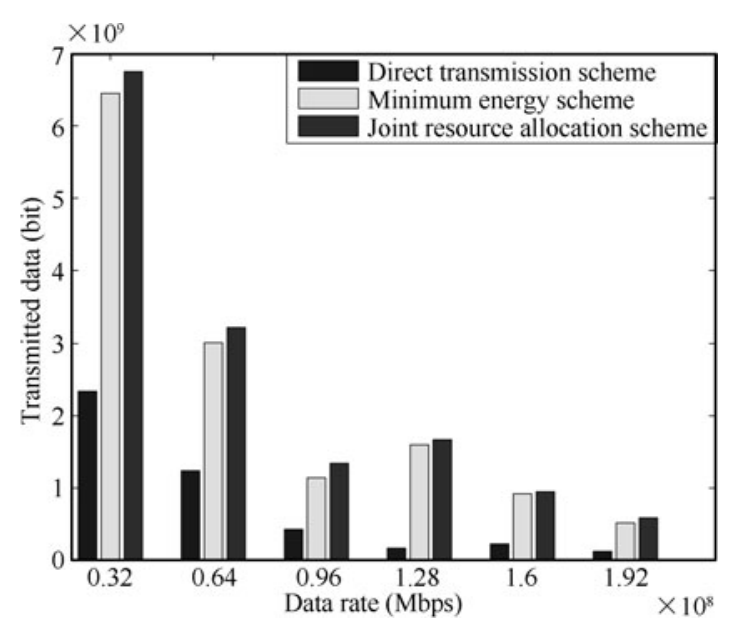

Fig. 3 Total transmitted data versus total data rate

\section{References}

[1] Y. Li, W. B. Wang, J. Kong, M. G. Peng. Subcarrier pairing for amplify-and-forward and decode-and-forward OFDM relay links. IEEE Communications Letters, vol. 13, no. 4, pp. 209-211, 2009.

[2] O. Duval, Z. Hasan, E. Hossain, F. Gagnon, V. K. Bhargava. Subcarrier selection and power allocation for amplifyand-forward relaying over OFDDM links. IEEE Transactions on Wireless Communications, vol.9, no. 4, pp. 12931297, 2010.

[3] W. B. Dang, M. X. Tao, H. Mu, J. W. Huang. Subcarrierpair based resource allocation for cooperative multi-relay OFDM systems. IEEE Transactions on Wireless Communications, vol. 9, no. 5, pp. 1640-1649, 2010.

[4] C. N. Hsu, H. J. Su, P. H. Lin. Joint subcarrier pairing and power allocation for OFDM transmission with decode-andforward relaying. IEEE Transactions on Signal Processing, vol. 59, no. 1, pp. 399-414, 2011.

[5] S. Kadloor, R. Adve. Relay selection and power allocation in cooperative cellular networks. IEEE Transactions on Wireless Communications, vol. 9, no. 5, pp. 1676-1685, 2010.

[6] K. Vardhe, D. Rennolds, B. D. Woerner. Joint power allocation and relay selection for multiuser cooperative communication. IEEE Transactions on Wireless Communications, vol. 9, no. 4, pp. 1255-1260, 2010.

[7] H. Hui, S. H. Zhu, G. M. Lv. Relay selection for lifetime extension in amplify-and-forward cooperative networks. In Proceedings of International Conference on Communications, IEEE, Cape Town, South Africa, pp. 1-5, 2010.

[8] F. Ke, S. L. Feng, H. C. Zhuang. Relay selection and power allocation for cooperative network based on energy pricing. IEEE Communications Letters, vol. 14, no. 5, pp. 396-398, 2010. 
[9] W. J. Huang, Y. W. P. Hong, C. C. J. Kuo. Lifetime maximization for amplify-and-forward cooperative networks. IEEE Transactions on Wireless Communications, vol.7, no. 5, pp. 1800-1805, 2008.

[10] M. Hajiaghayi, M. Dong, B. Liang. Maximizing lifetime in relay cooperation through energy-aware power allocation. IEEE Transactions on Signal Processing, vol. 58, no. 8, pp. 4354-4366, 2010.

[11] F. Z. Zuo, M. Dong. Prediction-based energy-aware relay cooperation for lifetime maximization. IEEE Wireless Communications Letters, vol. 1, no. 4, pp. 352-355, 2012.

[12] H. W. Khun. The Hungarian method for the assignment problem. Naval Research Logistics (NRL), vol.52, no. 1, pp. 7-21, 2005.

[13] Z. K, Sheng, J. G. Andrews, B. L. Evans. Adaptive resource allocation in multiuser OFDM systems with proportional rate constraints. IEEE Transactions on Wireless Communications, vol. 4, no. 6, pp. 2726-2737, 2005.

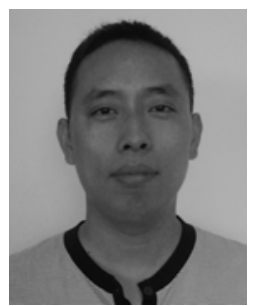

Chao-Ping Guo graduated from $\mathrm{Xi}^{\prime}$ an Communication Institute, China in 1997. He received the M.Sc. degree from Air Force Engineering University, China in 2003. He is a Ph. D. candidate at Xidian University, China. He is with $\mathrm{Xi}^{\prime}$ an Communication Institute now.

His research interests include routing, dynamic resource management, D2D comin wireless networks. munications and cooperative transmission

E-mail: chpguo@gmail.com (Corresponding author)

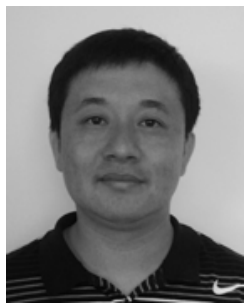

Kun Han graduated from Xi'an Communication Institute, China in 2002. He received the M.Sc. degree from Jiangnan University, China in 2005. He is a Ph. D. candidate at Xidian University. He is with $\mathrm{Xi}^{\prime}$ an Communication Institute now.

His research interests include crosslayer optimization, orthogonal-frequencydivision multiplexing (OFDM) and cooperative transmission.

E-mail: Hankunhk@163.com

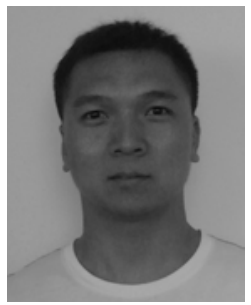

Xiao-Long Zhang graduated from People Liberation Army Information Engineering University, China in 2007. He received the M.Sc. degree from Xidian University, China in 2010, and is a Ph. D. candidate at Xidian University China.

His research interests include cross-layer optimization and carrier tracking.

E-mail: xiaolong12163@126.com

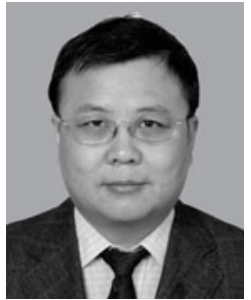

Hai-Lin Zhang graduated from Northwestern Polytechnic University, China in 1985. He received the M.Sc. degree from Northwestern Polytechnic University, China in 1988, and Ph. D. degree from Xidian University, China in 1991. He is currently the dean of School of Telecommunications Engineering at Xidian University and a key member at State Key Laboratory of Integrated Services Networks now.

His research interests include key transmission technologies and standards on broadband wireless communications for B3G, $4 \mathrm{G}$, and next-generation broadband wireless access systems.

E-mail: hlzhang@xidian.edu.cn 